\title{
Correlative Zernike phase contrast X-ray nanotomography to determine the distribution and orientation of graphite particles in a carbon fiber reinforced epoxy resin for improved thermal conductivity
}

Simon Carl ${ }^{1}$, Silvan Englisch ${ }^{1}$, Janis Wirth ${ }^{1}$, Benjamin Apeleo Zubiri ${ }^{1}$, Simon Bard ${ }^{2}$, Volker Altstädt ${ }^{2}$ and Erdmann Spiecker ${ }^{1}$

${ }^{1}$ Institute of Micro- and Nanostructure Research (IMN) \& Center for Nanoanalysis and Electron Microscopy (CENEM), Interdisciplinary Center for Nanostructured Films (IZNF), Department of Materials Science and Engineering, Friedrich-Alexander University Erlangen-Nürnberg (FAU), Erlangen, Germany, Erlangen, Bayern, Germany, ${ }^{2}$ Department of Polymer Engineering, University of Bayreuth, Germany, Bayreuth, Bayern, Germany

X-ray nanotomography (Nano-CT) is ideally suited for non-destructive three-dimensional (3D) imaging of carbon-based material systems on the nanoscale. The lab-based ZEISS Xradia 810 Ultra X-ray microscope enables standard absorption contrast imaging as well as edge-enhancing phase contrast imaging for low-Z materials by inserting a Zernike phase ring into the beam path behind the sample and Fresnel zone plate. While absorption contrast is usually employed for materials containing regions of sufficiently different atomic numbers, Zernike phase contrast allows to distinguish between different phases in composite materials and accentuates the morphology of embedded particles of similar density. The high-resolution and flexible imaging modes of the Nano-CT instrument, combined with scanning electron microscopy (SEM), allow for a detailed 3D study of the orientation and distribution of graphite particles in a representative $3 \mathrm{D}$ volume.

Light and resilient materials play an essential role in the manufacturing of novel electric turbines for airplanes. Likewise, thermal conductivity is one of the crucial properties of the turbine's shell that must be optimized before operational use. We investigated a promising material for the shell: carbon fiber and graphite particle reinforced composites. Besides the mechanical properties, the thermal conductivity is significantly improved by incorporating graphite platelets in between layers of unidirectional carbon fibers [1]. To achieve this, the carbon fibers are embedded in a graphite platelet enriched epoxide resin during a rolling manufacturing process. The platelet size averages at $4 \mu \mathrm{m}$ and the volume ratio composes up to 15 $\operatorname{vol} \%[1]$.

In particular, the local distribution and orientation of the graphite particles impact their interconnectivity and therefore influence the thermal conductivity. We acquired several Nano-CT tilt-series in large-fieldof-view (LFOV) phase contrast mode $(150 \mathrm{~nm}$ spatial resolution, $64 \mu \mathrm{m}$ field of view (FOV)) to determine the 3D distribution and relative orientation of the graphite platelets towards the carbon fibers in detail. The corresponding pillar sample with a diameter of $120 \mu \mathrm{m}$ was prepared by site-specific laser ablation using a 3D-Micromac microPREP PRO system. The combination of eight slightly overlapping LFOV tilt series (Figure 1b) enlarges the reconstructable volume and especially improves its statistical significance concerning the local variations in particle orientation and distribution. A smaller pillar of $10 \mu \mathrm{m}$ in diameter was site-specifically prepared from the larger pillar by focus ion beam (FIB) milling in a DualBeam FEI Helios NanoLab 660 to examine the particle interconnectivity of close-packed particles at a higher resolution. The particle size and morphology were examined on the milled surface by SEM imaging and in the pillar volume by high-resolution (HRES) phase contrast Nano-CT with $50 \mathrm{~nm}$ spatial resolution (Figure 1c inset). These measurements reveal that the particles indeed have a flat platelet morphology and already indicate, on the small scale, that there is a certain alignment of the the platelets along the carbon 
fibers. We further employed the HRES Nano-CT reconstruction to obtain an improved HRES-informed segmentation of the LFOV reconstruction, which was further supported by a machine-learning segmentation routine in the software Arivis Vision4D [2]. This routine enables us to significantly improve 3D data interpretation reliability of extended LFOV Nano-CT reconstructions by reducing segmentation errors caused by, for instance, adjacent particles and remaining Zernike phase contrast halo artifacts.

The applied segmentation routine allows for a detailed analysis of the orientation of the platelet particles with respect to the carbon fibers and the rolling plane of the manufacturing process. To accomplish this, the segmented platelets are projected onto the yz- (Figure 2a), xz- (Figure 2b) and xy-plane (not shown) of the reconstructed volumes, respectively. The $\mathrm{x}$-axis is set parallel to the fiber direction, which is also the rolling direction, and the xy-plane represents the rolling plane. The 3D orientation of the particles is determined by the Feret diameter of their projection onto a certain plane (i.e., the longest distance between any two points of one projected particle circumference) as indicated in the sketches in Figure 2a and $2 \mathrm{~b}$. The corresponding Feret angle describes the angle between the Feret diameter vector and the horizontal axis in the projected plane (here: the y-axis in the yz-plane and the X-axis in the xz-plane). This analysis reveals that the particle orientation correlates with the carbon fiber orientation and the anisotropic manufacturing process by compressing and rolling. The platelets arrange in one plane along the fiber long axis and rolling direction (x-axis) and the rolling plane (xy-plane) of the manufacturing process (Figure 2), which leads to a higher interconnectivity between the particles than randomly oriented particles and a better system-level electrical and heat-conducting properties of the material in a specific desired direction [1].

In conclusion, we applied comprehensive and scale-bridging HRES and extended LFOV Nano-CT complemented by SEM imaging to investigate the relationship between manufacturing process, 3D particle orientation and material properties. This correlative microscopic analysis unveils the reason for the outstanding thermal properties of this carbon fiber reinforced composite material in a deterministic way. This knowledge over the relationship between material properties, manufacturing process and the 3D structure supports further targeted optimization of this composite material.
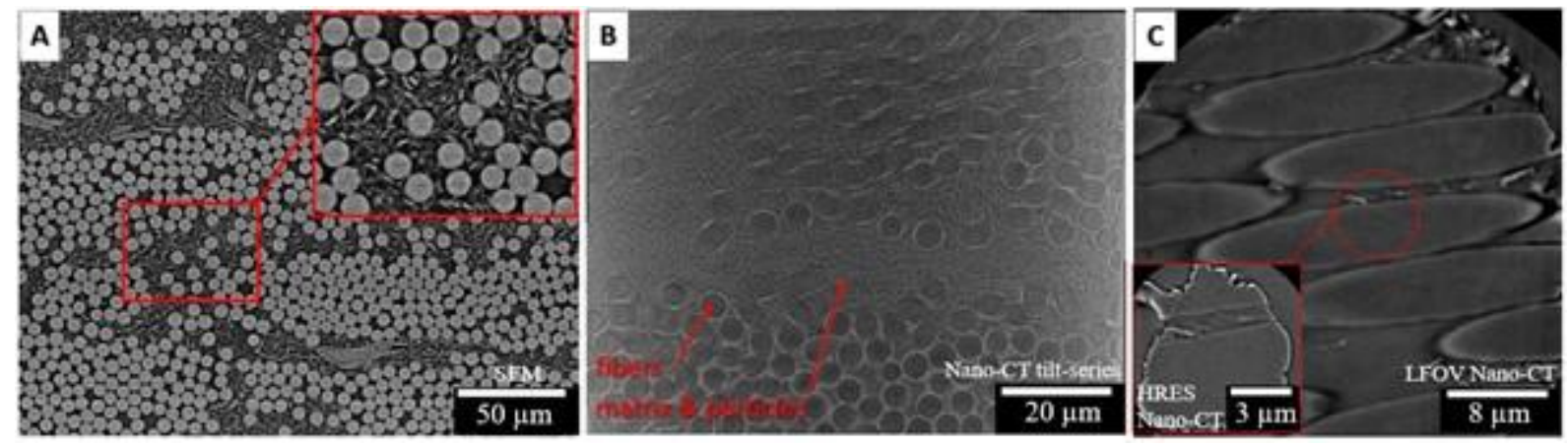

Figure 1. Correlative SEM and Nano-CT investigation. (a) SEM image of the investigated material system. The carbon fibers with a mean diameter of roughly $7 \mu \mathrm{m}$ can be seen along its long axis in this cross-sectional image. The graphite particles are embedded in the resin matrix between the fibers. (b) Nano-CT projection from a tilt series composed of eight adjacent stitched LFOV phase contrast images at the same tilt angle featuring the carbon fiber structures oriented orthogonally to the image plane. (c) Virtual slice through a single LFOV reconstruction. The carbon fibers are visible as oval-shaped structures since they are diagonally cut in this view. The inset shows a virtual slice through a HRES Nano-CT reconstruction of a site-specifically prepared, smaller pillar to investigate the graphite particle size and morphology accurately and further use it to improve the segmentation quality of the LFOV reconstructions. 

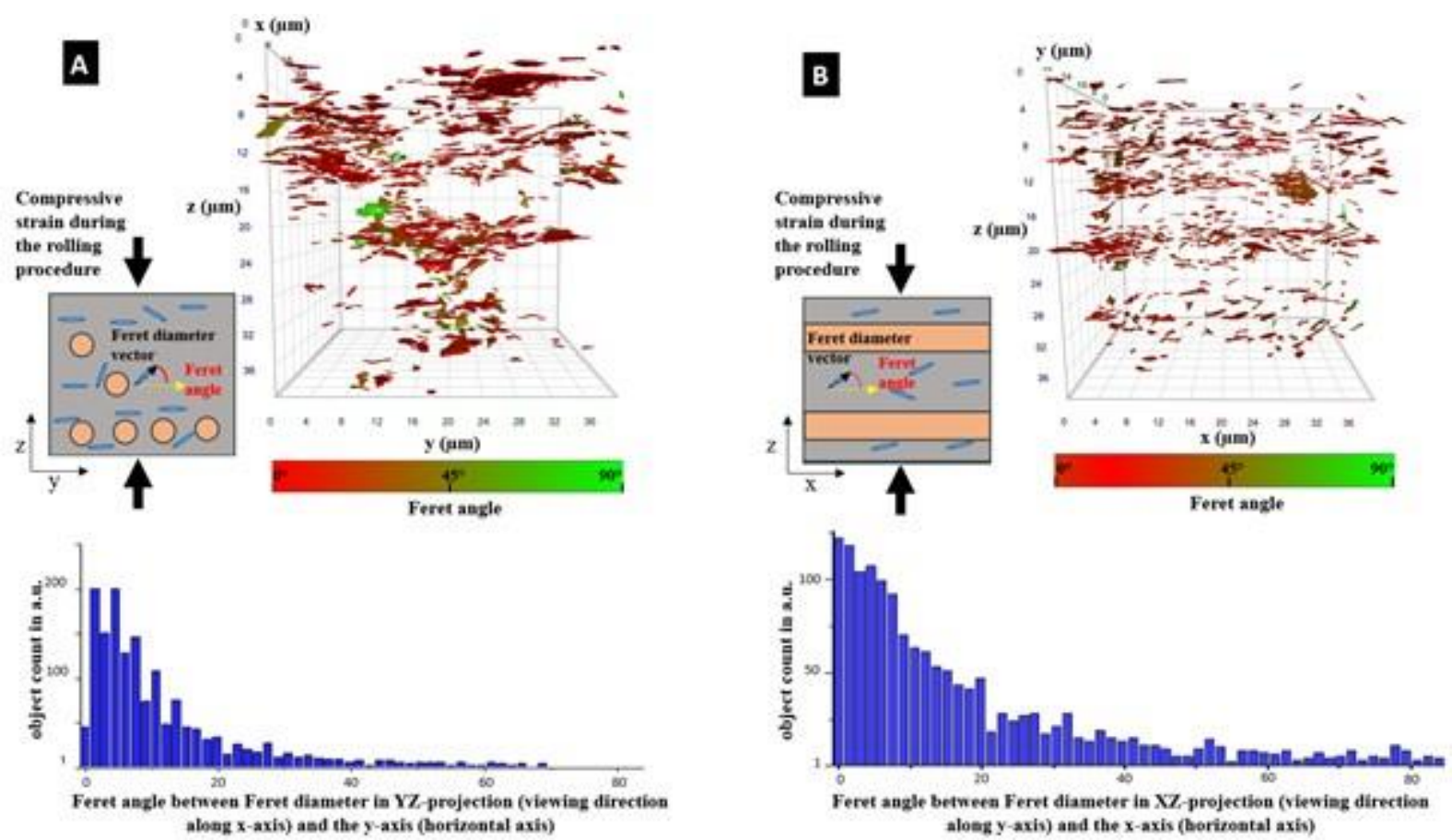

Figure 2. Analysis of the particle orientation with respect to the carbon fibers, determined by the projected Feret diameter vector in the yz- and xz-plane, respectively - 3D representation of a single segmented LFOV Nano-CT reconstruction with the carbon fibers aligned along the x-axis: representation with the Feret angle (a) between the projected Feret diameter onto the yz-plane and the y-axis and (b) between the projected Feret diameter onto the xz-plane and the x-axis. The red color-coding indicates a parallel orientation of the platelets towards the respective axis. The platelets preferably arrange parallel along the fiber direction (x-axis) and further orient parallel to the xy-plane, which is also the rolling plane of the manufacturing process, as represented in the Feret angle distribution plots. This leads to a better connectivity of the graphite particles in the rolling plane and the increased thermal conductivity.

\section{References}

[1] Bard, et al., Transverse Thermal Conductivity of Epoxy Carbon Fiber Prepreg Laminates with a Graphite Filled Matrix. J. Compos. Sci., 2019. 3(2), p. 44.

[2] C. Sommer, C. Straehle, U. Köthe, F. A. Hamprecht, Ilastik: interactive learning and segmentation toolkit. IEEE International Symposium on Biomedical Imaging: From Nano to Macro, 2011.

\section{Acknowledgment:}

The authors thank the DFG for financial support within the frameworks of the research training group GRK1896 "In situ Microscopy with Electrons, X-rays and Scanning Probes", the project SP648/8 "Highresolution X-ray microscopy for correlative tomography, high throughput screening and in situ mechanical testing of structural and functional materials." and the Collaborative Research Centre 1411 "Design of Particulate Products" (Project-ID 416229255). 